\title{
Diagnostics and Upgrade of the DAFNE Beam Test Facility (BTF)
}

\author{
P. Valente ${ }^{\mathrm{a}}$, B. Buonomo ${ }^{\mathrm{b}}$ and G. Mazzitelli ${ }^{\mathrm{b}}$ \\ ${ }^{a}$ INFN Sez. di Roma, \\ P.le A. Moro 2, I-00185 Roma, Italy \\ ${ }^{b}$ Laboratori Nazionali di Frascati dell'INFN, \\ Via E. Fermi 40, I-00044 Frascati (RM), Italy
}

The DAFNE Beam Test Facility (BTF), operational in Frascati LNF since November 2002, is a beam transfer line optimized for single particle production, mainly for high-energy detectors calibration. It can provide $e^{-} / e^{+}$ beams in a wide range of intensity - between single particle and $10^{10}$ particles/pulse - and energy, from few tens of $\mathrm{MeV}$ up to $800 \mathrm{MeV}$; the pulse duration can also be adjusted between 1 and $10 \mathrm{~ns}$ at a maximum repetition rate of $50 \mathrm{~Hz}$. In the last year many groups have accessed the facility, for testing a number of apparata: silicon detectors, calorimeters, scintillator counters, GEM (in single particle mode); fluorescence counters, crystal and nanotube bendings, Cerenkov counters, thermo-acoustical detectors (at high intensity). The large beam multiplicity range required to implement different diagnostic devices: calorimeters for particle counting at low intensity, fluorescence chambers and Cerenkov detectors for intermediate-high intensity monitor, up to standard beam diagnostic systems. The beam spot profile and position is also measured by means of a $x-y$ scintillating fiber hodoscope with multi-anode PMT readout.

\section{The DAFNE BTF beam}

The DAFNE Beam Test Facility (BTF), operational in Frascati LNF since November 2002, is a beam transfer line optimized for the production of a pre-determined number of electrons or positrons. It can provide electron or positron beams in a wide range of intensity, between single particle up to $10^{10}$ particles/pulse (now running at lower intensity, since only $10^{3} / \mathrm{s}$ are allowed); and energy, from few tens of $\mathrm{MeV}$ up to $800 \mathrm{MeV}$. The pulse width can also be changed, either 1 or $10 \mathrm{~ns}$, at a maximum repetition rate of $50 \mathrm{~Hz}$.

The facility is particularly suitable for particle detector testing purposes, such as energy calibration and efficiency measurement, in single electron mode; while beam diagnostics devices and detectors can be studied at high intensities. The BTF is a part of the DAFNE accelerator, consisting of a double ring electron-positron collider, a high current linear accelerator (Linac), an intermediate damping ring and a system of $180 \mathrm{~m}$ transfer lines connecting the whole system. The $\mathrm{e}^{+} / \mathrm{e}^{-}$beam produced by the LINAC is stacked and damped in the accumulator ring, from which it is extracted and injected in the main rings. When the injector system is not delivering beams to the accumulator, the LINAC beam, suitably attenuated, can be trasported into the beam test area by a transfer line (BTF line).

During BTF operation, the high current Linac beam is attenuated by a variable depth target in order to strongly increase the energy spread of the primary beam; then the emergent particles are energy selected by means of a bending magnet and slits system. The energy selector only accepts a small fraction of the resulting energy distribution, thus reducing the number of particles in the beam by a large and tunable factor.

In the BTF, the most effective way to change the average number of particles in the beam is to change the selected energy $E_{\mathrm{sel}}$; in particular, at the same Linac energy and intensity and with the same collimator settings, the multiplicity increases by decreasing the chosen $E_{\text {sel }}$.

In addition, the multiplicity can be tuned by changing the aperture of the upstream and/or 
downstream collimators; in particular, the measured multiplicity increases by increasing the slits aperture until the intrinsic beam spot size is exceeded.

The dipole magnet and slits system determine the relative energy spread, from the effect of the momentum dispersion introduced by bending magnet. In the standard BTF operation for a wide range of slits aperture a resolution better of $1 \%$ can be obtained.

Further details on the BTF characteristics, on the single particle production and on the beam optimization can be found in Ref. [1].

\section{Beam profile detector}

In order to improve the diagnostics of the BTF facility, a scintillating fiber hodoscope was designed, constructed, tested and put in operation during year 2003, in order to monitor the beam spot size and position with millimetric accuracy in real-time[2].

Since high-fluorescence metallic flags are not sensitive at very low beam intensities, expecially for the single particle operation mode, a position sensitive particle detector was chosen. The detector was conceived keeping in mind the wide range of beam conditions (in energy and multiplicity), the typical beam spot characteristics (with an optimum size of $2 \times 2 \mathrm{~mm}^{2}$ ), and the need for a nondestructive, easy to implement and compact detector.

The hodoscope is composed by two stacks of $1 \mathrm{~mm}$ diameter cladded scintillating fibers (Pol.Hi.Tech 0046), to have the $x$ and $y$ beam profile. Each stack is composed by four layers, staggered by half fiber diameter, in order to minimize dead-zones and to have a good light yield even with a non-optimal coupling to the photocathode. Each view of the two views is composed by 16 bundles of three aligned four-layers fiber stacks, for a total active area of $48 \times 48 \mathrm{~mm}^{2}$; the fibers are glued with optical cement BICRON BC600. Each bundle of fibers is then coupled, by means of a grooved PVC mask, to the surface of a 16 pixels $\left(4.2 \times 4.2 \mathrm{~mm}^{2}\right.$ each $)$ multi-anode photomultiplier tube (Hamamatsu H6568), operated at a typical gain of $2 \times 10^{6}$. The multi-anode PMT analog signals are read-out by a charge-integrating ADC, $12 \mathrm{bit}, 0.1 \mathrm{pC} /$ count, with a $100 \mathrm{~ns}$ gate generated starting from the Linac gun timing system (details on the BTF DAQ system are reported in Ref. [3]).
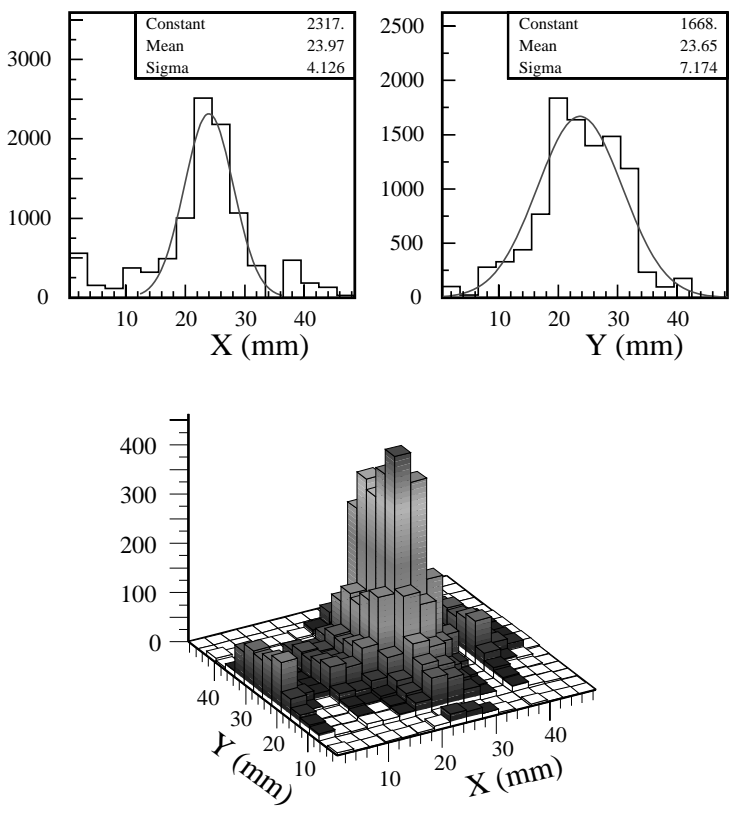

Figure 1. Charge-weighted distributions for $x$ and $y$ planes of the scintillating fibers detector (top), and corresponding two-dimensional beam spot (bottom); focussed beam, $493 \mathrm{MeV}$ electrons.

In order to get the beam profile, the pulse height of the 16 multi-anode PMT channels, pedestal subtracted, are accumulated in an histogram. The shape of the resulting chargeweighted profile is directly related to the beam spot. An example of the beam profile transverse distribution, obtained with the two detectors mounted with the fibers running along the horizontal and vertical directions is shown in Fig. 1, for a well focussed, $493 \mathrm{MeV}$, electron beam, with an average of 1-2 particles per pulse. 
The shape of the beam spot is fairly Gaussian, and the beam dimensions are in good agreement with the beam-spot measurements with similar beam conditions, during a campaign with a silicon strip detector (in collaboration with the AGILE group).

The measured $x$ and $y$ profiles are displayed online in the BTF control system: 100 events are sufficient to get a fairly good beam profile (at least for a focussed beam); this translates in a refresh time of 4 seconds at $50 \mathrm{~Hz}$ repetition rate.

\section{Cerenkov detector}

The more convenient way of counting particles in the BTF beam at low multiplicity, and in particular in the single particle mode, is the calorimetric measurement: the total energy deposited in a lead/scintillating fiber calorimeter is proportional to the number of electrons/positrons of a given energy. This is a very effective multiplicity estimate, provided that the beam energy is above $\approx 100 \mathrm{MeV}$, since the calorimeter resolution is at the level of $9 \%$ at $500 \mathrm{MeV}$.

However, above $\approx 20$ particles this calorimetric method becomes no longer effective due to saturation effects.

In order to have a diagnostic device in the $\mathrm{n}=$ 100-1000 range (and higher), a different detector has been developed and tested in collaboration with the AIRFLY group [4]. It is essentially a counter based on the Cerenkov light emission in a PLEXIGLAS radiator when crossed by relativistic electrons (which in the BTF energy range is always the case).

The light is then extracted by properly shaping the end part of the radiator itself and collected by a photomultiplier, without optical connection: this gives the possilibity of interposing a calibrated optical lter between the radiator and the PMT, in order to attenuate the Cerenkov light by a known factor, thus extending the dynamical range of the counter.

The Cerenkov light yield, and in turn the phototube analog signal, should be proportional to the number of electrons crossing the radiator; this phenomenon should be linear up to a very high number of electrons.
The Cerenkov counter signal shows a good correlation with the energy deposited in the calorimeter, as shown in Fig. 2, with a suitably low multiplicity beam; using the cross-calibration between the two detectors, the Cerenkov counter has been used to monitor the beam multiplicity up to $\approx 1000$.
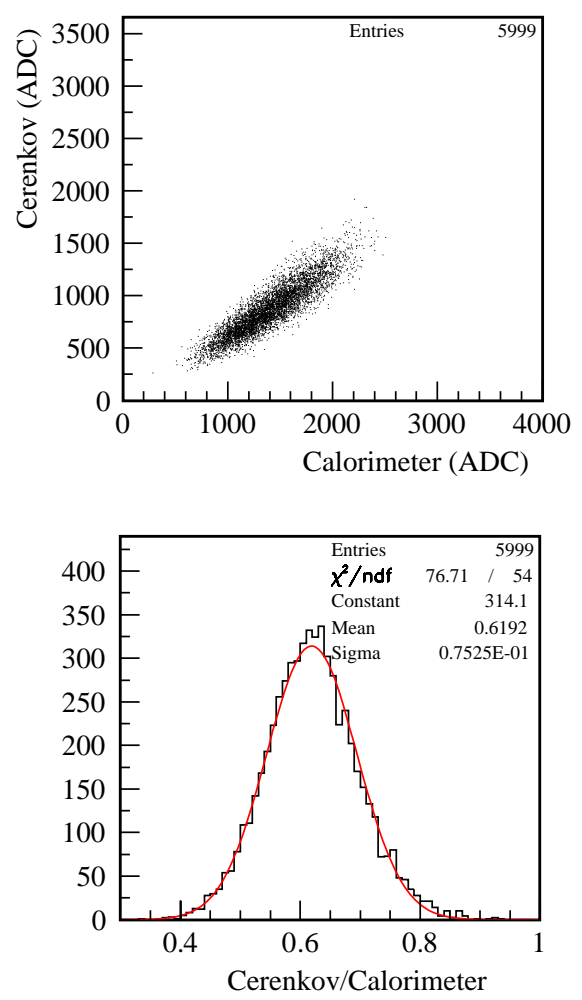

Figure 2. Correlation between the pulse height in the Cerenkov counter and in the total absortion calorimeter (top) and ratio of the two (bottom), with Gaussian fit.

\section{2004 BTF upgrade}

The availability of the BTF beam was limited during the runs in the period 2002-2003 by the main DAFNE experiments, KLOE and FINUDA. 
The KLOE detector is able to take data also during the injection of the beams, practically leaving no time for BTF operation. During FINUDA operation the typical duty-cycle for the BTF was $\approx$ $50 \%$. In order to overcome these limitations, a complete separation between the DAFNE transfer lines to the main rings, and the BTF channel was planned [5]. In fact an independent transfer line for the BTF, only gives a dead-time connected to the Linac switching time and the time spent for filling the main rings.

The chosen solution for the upgrade of the facility is to have a triple exit: a straight line towards the accumulator (and from there to the main rings); the spectrometer line at $7^{\circ}$, dedicated to the Linac beam energy measurement, to which a single shot per injection cycle is sent; and finally an exit at $3^{\circ}$ for the BTF channel. To minimize the time of switching, the ideal solution would have been a variable pulsed magnet. For the first phase the existing pulsed magnet is used to send the single pulse per cycle to the spectrometer line, as in the past, while for the BTF channel a fast dipole can be switched in a few seconds. The final portion of the Linac has been completely redesigned in order to match these modifications.

Moreover the attenuating target and selector system (dipole magnet+slits) has been moved on the BTF dedicated trasfer-line. All the modifications to vacuum pipes, magnets and services were completed; the new lines have been successfully commissioned, and the BTF has restarted beam delivery from May 2004.

\section{Conclusions}

The DAFNE Beam Test Facility showed very good performances, both from the point of view of operation reliability and of the flexibility in order to answer very different experimental needs: electron and positron beams were produced in the energy range from 50 to $700 \mathrm{MeV}$, in single particle mode as well as in at high intensity. Moreover, the beam diagnostics was effectively exploited both to monitor the beam conditions, and to adjust them to the experimental requests. Finally, the recent upgrade of the facil- ity, with a dedicated beamline for the BTF, allows to significantly improve the duty-cycle and to futher increase the beam time dedicated to the user groups.

\section{REFERENCES}

1. G. Mazzitelli, A. Ghigo, F. Sannibale, P. Valente and G. Vignola, Nucl. Instum. Meth. A 515 (2003) 516.

2. M. Anelli, B. Buonomo, G. Mazzitelli and P. Valente, A scintillating-fiber beam profile monitor for the DAFNE BTF, LNF report (2004).

3. G. Mazzitelli and P. Valente, Commissioning of the DAFNE Beam Test Facility, LNF report LNF-03-003(P) (2003).

4. G. Mazzitelli, F. Sannibale, P. Valente, M. Vescovi, P. Privitera and V. Verzi, Beam Instrumentation for Single Electron DAFNE Beam Test Facility, Proc. of DIPAC 2003.

5. G. Mazzitelli, M.A. Preger, C. Sanelli, F. Sgamma and P. Valente, DAFNE Beam Test Upgrade Proposal, DAFNE Technical note BTF-1 (2003). 